\title{
FAR-ULTRAVIOLET SURVEYS OF GLOBULAR CLUSTERS: SEARCHING FOR THE PRODUCTS OF STELLAR COLLISIONS AND NEAR MISSES
}

\author{
C. Knigge, ${ }^{1}$ D. R. Zurek, ${ }^{2}$ M. M. Shara, ${ }^{2}$ K. S. Long, ${ }^{3}$ R. L. Gilliland,${ }^{3}$ and P. A. Charles ${ }^{1}$
}

\section{RESUMEN}

Las observaciones en el ultravioleta lejano (FUV) con el Telescopio Espacial Hubble (HST) proporcionan un excelente camino para encontrar y estudiar las poblaciones de estrellas azules y calientes en los núcleos de cúmulos globulares. Estias poblaciones incluyen "stragglers" azules formadas dinámicamente y binarias que interactúan entre sí (tales comı variables cataclísmicas, es decir, los productos de colisiones estelares y de cuasi-colisiones). Utilizando el cúmulo 47 Tuc como un ejemplo, mostramos cómo la combinación de imágcnes FUV y de espectroscopía sin rendija pueden utilizarse para descubrir y estudiar estas poblaciones.

\section{ABSTRACT}

Far-ultraviolet (FUV) observations with the Hubble Space Telescope are an excellent way to find and study the hot, blue stellar populations in the cores of globular clusters. These populations include dynamicallyformed blue stragglers and interacting binaries (such as cataclysmic variables, i.e. the products of stellar collisions and near misses. Using the cluster 47 Tuc as an example; we show how the combination of FUV imaging and slitless spectroscopy can be used to uncover and study these populations.

Key Words: GALAXY: GLOBULAR CLUSTERS: INDIVIDUAL (47 TUCANAE) - NOVAE. CATACLYSMIC VARIABLES — STARS: BLUE STRAGGLERS — ULTRAVIOLET: GENERAL - WHITE DWARFS

\section{INTRODUCTION}

Globulai clusters (GCs) are fantastic stellar crash test laboratories. Violent encounters between binaries and single stars in dense cluster cores give rise to exotic stellar populations, such as blue stragglers (BSs), cataclysmic variables (CVs) and low-mass Xray binaries. All of these objects have considerably bluer spectral energy distributions than "normal" cluster members. CVs and young white dwarfs (WDs), in particular, radiate much of their luminosity in the far-UV. Observations in this waveband are therefore well suited to the detection and characterization of these important stellar species. We have therefore embarked on a program to study the cores of GCs at FUV wavelengths, using both imaging and slitless spectroscopy with STIS and ACS onboard HST. Here, we present results from observations of our first target, 47 Tucanae (for details, see Knigge et al. $2003 ; 2003$ )

\section{RESULTS}

Figure 1 shows a comparison of FUV (STIS/F25QTZ; a bandpass centered around $1600 \AA$ ) and U-band (WFPC2/F336W) images

\footnotetext{
${ }^{1}$ University of Southampton, UK.

${ }^{2}$ American Museum of Natural History, USA

${ }^{2}$ Space Telescope Science Institute, USA.
}

of the cluster core. The tremendous difference in crowding between these images nicely illustrates the ease with which hot stellar populations can be detected in the FUV. The color-magnitude diagram produced from this data is shown in Figure 2.

As discussed in Knigge et al. (2002), the FLV imaging data has already allowed us to: (i) find $\mathrm{FCV}$ counterparts to all of the Chandra Cl cindidates in the core of this cluster; (ii) confirm the ( $\mathrm{T}$ nature of these candidates based on variability and location in the far-UV colour-magnitude diagram: (iii) suggest several additional CV candidates: (iv) detect a clean, well-populated far-UV BSS sequence: $(v)$ detect numerous young WDs on the upper end of the cooling curve.

Since crowding is not a serious problem in the FUV, it is possible to carry out multi-object slitles: spectroscopy even in the dense core of a globular cluster like 47 Tuc. This is an ext remely efficient way of spectroscopically confirming interesting sunres. such as CV candidates. The power of this approach is demonstrated in Figure 3, which shows the slitless 2-D spectral image of 47 Tuc and the 1-D extracted spectrum of the brightest source. the eclipsing binary AkO 9. The strong $\mathrm{C}$ IV and He II emission line's in immediately suggest that $\mathrm{AhO} g$ is a catinclysmic variable (see Knilgge et al. 2003). 

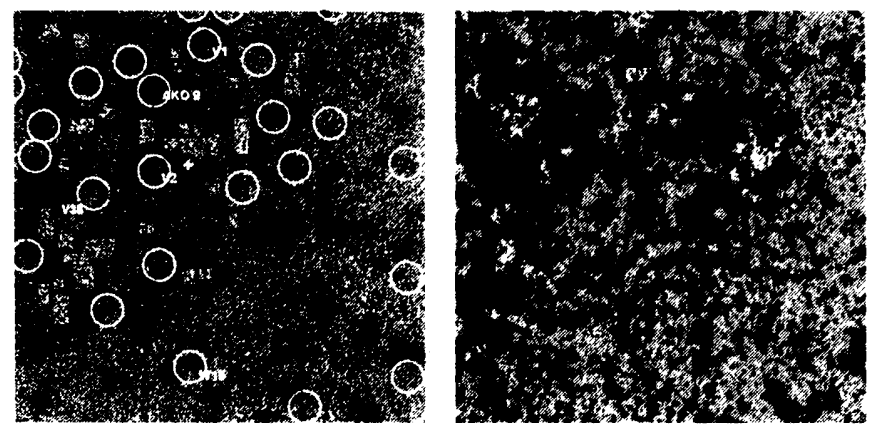

Fig. 1. Left Panel: A $25^{\prime \prime} \times 25^{\prime \prime}$ far-UV image of the core of 47 Tuc. The cluster center is marked as a cross. The positions of previously known blue objects (squares), Chandra $x$-ray sources (big circles) and CV candidates (small circles) arr marked. The four confirmed CVs within the FUV field are labelled with their names. Right Panel: The WFPC2/F336W image of the same field. The difference in crowding is obvious.

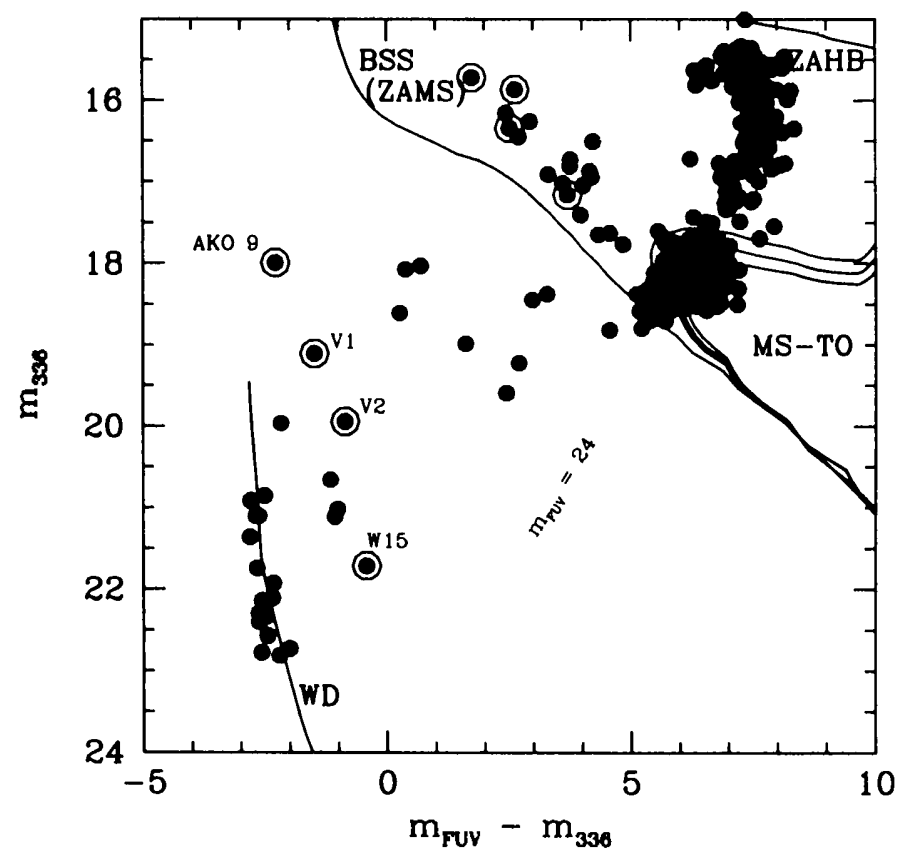

Fig. 2. The FUV/optical color-magnitude diagram derived from the data in Fig. 1. Variable FUV sources are marked with black circles, and the four previously known or suspected $\mathrm{CVs}$ are labeled with their names. The diagonal line marks the FUV completeness limit. The other lines in the diagram indicate the expected locations of various stellar populations. I'mmarked sources between the WD and main sequences are potential new $\mathrm{CV}$ candidates.
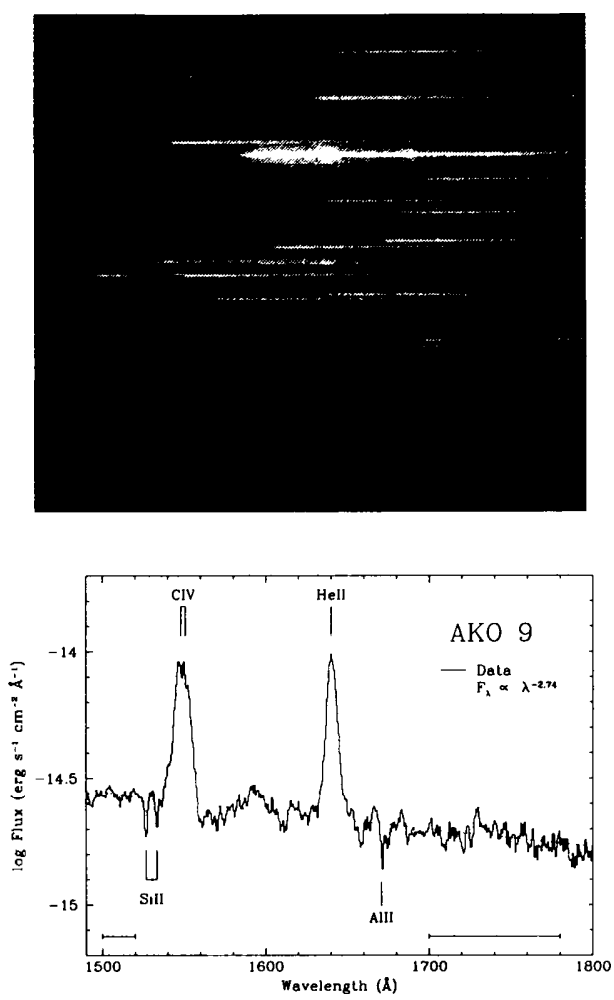

Fig. 3. Top Panel: The 2-D spectral image obtained from our slitless FUV spectroscopy of the field shown in Fig. 1. The brightest source is the cataclysmic variable AKO 9, which shows clear evidence for line emission. Bottom Panel: The extracted 1-D FUV spectrum of AKO 9 (data affected by eclipses was excluded).

\section{CONCLUSIONS \& THE FUTURE}

Far-ultraviolet surveys are an excellent tool for studying the most interesting hot stellar populations in globular clusters, including the dynamicallyformed interacting binaries. Here, we have presented a snapshot of our work in this area, but there is much more to come. For example, we already have preliminary spectroscopic confirmation of two additional CV candidates in 47 Tuc, FUV observations of several other clusters are being analysed, and additional observations are already scheduled. Watch this space!

\section{REFERENCES}

Knigge, C., Zurek, D. R., Shara, M. M., \& Long, K. S. 2002, ApJ, 579, 752

Knigge, C., Zurek, D. R., Shara, M. M., Long, K. S., \& Gilliland, R.L. 2003, ApJ, 599, 1320

P. A. Charles and C. Knigge: School of Physics \& Astronomy, University of Southampton, Southampton SO17 1B.J. UK (pac (lastro.soton.ac.uk.christianciastro.soton.ac.uk).

R. T. Gilliland and K. S. Long: Space Telescope Science Institute, 3700 San Martin Drive, Baltimore, MD 21218 (gillilastsci.edu, long(1stsci.edu).

M. M. Shara and D. R. Zurek: Ancricinl Museum of Natural History. 79th Street and Central Park West, New York, NY 10024 (msharaOamnlı.org. dzurek (ammul.org). 